\title{
A REMARK ON THE CARDINAL OF LIMIT SPACES
}

\author{
WALTER S. SNYDER
}

In this note we show that any nonvacuous set can be topologized to form a compact $L^{*}$ space, ${ }^{1}$ and thus the cardinal of such spaces is unrestricted.

If $S$ is normally ordered and the order has a last element we may define lim sup $\left\{a_{k}\right\}$ to be the first element of the order which precedes only a finite number of the terms of $\left\{a_{n}\right\}$, and $\lim \inf \left\{a_{n}\right\}$ to be the first element of the order which is preceded by, or equals, infinitely many terms of $\left\{a_{n}\right\}$. We term a sequence $\left\{a_{n}\right\}$ convergent if and only if $\lim \inf \left\{a_{n}\right\}=\lim \sup \left\{a_{n}\right\}$, and the common element is defined to be the limit. The reader will verify that this defines a compact $L^{*}$ space. Our result thus appears as a consequence of the well known Zermelo theorem to the effect that any set can be normally ordered.

Our result can be obtained, however, without the use of normal ordering, though the topologization is perhaps less natural.

Let $S$ be a given set and $x_{0}$ a fixed element of $S$. We define the class of convergent sequences together with their limits to consist of :

(a) All sequences $\left\{a_{n}\right\}$ for which $a_{n}=a$ for sufficiently large $n$, and the limit is $a$.

(b) All sequences $\left\{a_{n}\right\}$ in which $x_{0}$ is the only element of $S$ occurring infinitely often (including the case in which no element of $S$ occurs infinitely often), and the limit is defined to be $x_{0}$ in either case. It is easily verified that this defines a compact $L^{*}$ space in $S$.

Ohio State University

Received by the editors April 11, 1941.

${ }^{1}$ An $L^{*}$ space is a limit space in which certain sequences are designated as convergent, and a limit assigned to each in such a way that

(1) $a, a, a, \cdots$ converges to $a$.

(2) " $\left\{a_{n}\right\}$ converges to $a$ " implies "every subsequence of $\left\{a_{n}\right\}$ converges to $a$."

(3) If $\left\{a_{n}\right\}$ does not converge to $a$ there exists a subsequence of $\left\{a_{n}\right\}$, say $\left\{b_{n}\right\}$, such that no subsequence of $\left\{b_{n}\right\}$ converges to $a$. The space is compact if every sequence has a convergent subsequence. (See Kuratowski, Topologie, vol. 1, p. 76, or Fréchet, Les Espaces Abstraits.) 